\title{
Análisis comparado de los procesos de calidad en fisioterapia / kinesiología de Colombia y Chile
}

\author{
Comparative Analysis of the Quality Processes in Physiotherapy/Kinesiology of Colombia and Chile \\ Análise comparativa dos procesos de qualidade na fisioterapia / cinesiologia Colombia e Chile \\ Luis Fernando Rodríguez Ibagué $\mathrm{MPA}^{1}$, Andrés Felipe Sánchez Medina Ft', Paola Andrea Zamora Restrepo Ft', Luis \\ Alejandro Araya Veliz $\mathrm{MBA}^{2}$ \\ Recibido: 19 de febrero de $2014 \bullet$ Aceptado: 25 de febrero de 2015 \\ Doi: dx.doi.org/10.12804/revsalud13.02.2015.05 \\ Para citar este artículo: Rodríguez-lbagué LF, Sánchez-Medina AF, Zamora-Restrepo PA, Araya-Veliz LA. Análisis comparado de los procesos de calidad en fisioterapia / \\ kinesiología de Colombia y Chile. Rev Cienc Salud. 2015;13(2):187-204. doi: dx.doi.org/10.12804/revsalud13.02.2015.05
}

\section{Resumen}

Introducción: Las iniciativas de las reformas en América Latina enmarcan una preocupación por asegurar la cobertura universal y prestar servicios con eficiencia, por ende, la gestión de la calidad se convierte en uno de los temas más importantes en el siglo Xxi, en especial con temas relacionados con la salud. Objetivos: Caracterizar los procesos de habilitación / autorización sanitaria y acreditación de los servicios kinesiológicos de Colombia y Chile desde la perspectiva de la calidad en salud. Materiales y métodos: Para esto se realizó un análisis comparativo descriptivo entre países (Colombia y Chile) de los procesos de calidad en Kinesiología, lo que evidenció similitudes y diferencias relacionadas con el aseguramiento de la calidad. Discusión: Ambos países cuentan con estándares similares en cuanto Habilitación / Autorización sanitaria y Acreditación. Actualmente, en dichos países se discuten temas como seguridad del paciente, derechos y deberes de los pacientes, infraestructura, acceso, evaluación de la atención, talento humano, entre otros, todo en pro de garantizar la calidad del servicio que está en desarrollo actualmente y se ha venido incorporando a la cultura organizacional de los servicios. Conclusiones: En las revisiones de la literatura se evidencia poca documentación frente a los procesos de calidad, específicamente para kinesiología. Por ende, es importante proporcionar a la comunidad académica el análisis de los estándares requeridos para la Habilitación y Acreditación que contribuya al enriquecimiento del área de administración y gestión en la profesión.

Palabras clave: Kinesiología, calidad, acreditación, habilitación, salud.

\footnotetext{
1 Escuela de Medicina y Ciencias de la Salud, Universidad del Rosario. Correspondencia: Carrera 24 \# 63C-69. Correo electrónico: luisf. rodriguez@urosario.edu.co

2 Universidad Mayor (Chile).
} 


\begin{abstract}
Introduction: The initiatives in Latin American reforms frame a concern to ensure universal coverage and provide quality services, thus the quality management has become one of the most important issues in the $21^{\text {st }}$ century, especially in health issues. Objectives: To characterize the health habilitation/authorization and accreditation processes of kinesiological services in Colombia and Chile, from the perspective of health quality. Methodology: A descriptive comparative analysis between countries (Colombia and Chile) in terms of quality processes in Kinesiology was conducted, showing similarities and differences related to quality assurance. Discussion: Both countries have similar standards in terms of health habilitation/authorization and accreditation. Currently, in both countries issues are discussed such as patient safety, patient's rights and duties, infrastructure, access, care assessment, human talent, and others; all for the sake of ensuring service quality, that is currently developing and is been incorporated into the services organizational culture. Conclusions: Reviewing literature, poor documentation towards quality processes, specifically for kinesiology is evidenced. It is therefore important to provide to the academic community analysis of standards required for health habilitation and accreditation, thus contributing to enriching the administration and management area of our profession.
\end{abstract}

Keywords: Kinesiology, quality, accreditation, enabling and health.

\title{
Resumo
}

Introdução: As iniciativas das reformas na América Latina emolduram um a preocupação por assegurar a cobertura universal e emprestar serviços com eficiência, portanto a gestão da qualidade se converte em um dos temas mais importantes no século xxI, em especial com temas relacionados com a saúde. Obketivos: caracterizar os processos de habilitação/autorização sanitária e acreditação dos serviços Kinesiológicos da Colômbia e do Chile desde a perspectiva da qualidade em saúde. Materiais e métodos: Para isto se realizou uma análise comparativa descritiva entre países (Colômbia-Chile) respeito a processos de qualidade em Kinesiologia evidenciando similitudes e diferenças relacionadas com a garantia da qualidade. Discussão: Ambos os países contam com standards similares respeito a Habilitação/Autorização sanitária e Acreditação. Atualmente em ditos países discutem-se temas como segurança do paciente, direitos e deveres dos pacientes, infraestrutura, acesso, avaliação da atenção, talento humano, entre outros, todo em pro de garantir a qualidade do serviço que estão em desenvolvimento atualmente e se tem vindo incorporando à cultura organizacional dos serviços. Conclusões: Nas revisões da literatura se evidencia pouca documentação frente aos processos de qualidade especificamente para Kinesiologia. Portanto, é importante proporcionar à comunidade académica a análise dos standards requeridos para a Habilitação e Acreditação que contribua ao enriquecimento da área de administração e gestão em nossa profissão.

Palavras-chave: Kinesiologia, qualidade, acreditação, habilitação, saúde. 


\section{Introducción}

Las reformas del sector salud, en la mayoría de los países latinoamericanos, han mostrado favorecer la participación del sector privado en la gestión pública y la prestación del servicio, esto muestra una visión de libre mercado en el servicio público de la salud. Igualmente, otros aspectos comunes a las reformas son evidenciados por la puesta en práctica de descentralizar los establecimientos públicos y por la realización de contratos entre el sector público y privado con participación social. No obstante, actualmente las políticas de reformas a los servicios de salud en estos países asignan al Estado el rol de rectoría y regulador (1). En términos concretos, las iniciativas de las reformas enmarcan una preocupación por asegurar la cobertura universal y por prestar servicios con calidad, por ende, la gestión de la calidad se ha convertido en uno de los temas más importantes en el siglo xxI, en especial con temas relacionados con la salud (2).

Los sistemas de salud de Colombia y Chile son modelos de competencia gestionada que se asemejan en su financiación, ya que en los dos países los trabajadores hacen un aporte y realizan copagos, buscan la cobertura universal y, además, la rectoría y regulación se hace desde los ministerios. La prestación de los servicios para la población no asegurada en Colombia se hace por medio de la red pública y en Chile por medio del fonasa (Fondo Nacional de Salud). Los dos países tienen un sistema de calidad en el que el propósito es disminuir los riesgos en la atención para los usuarios. Para asegurar la calidad de la atención de los usuarios, los dos países tienen estándares de habilitación (Autorización Sanitaria) y Acreditación, que se aplican a los servicios de salud en general $y$, por consiguiente, a fisioterapia / kinesiología (3).

Los servicios de fisioterapia, en Chile como en Colombia, no son ajenos al impacto de las re- formas de la salud, donde se ve afectada la calidad en la atención de Salud, en cuanto a la duración de sesiones y al número de pacientes en estas. El profesional en fisioterapia, para lograr tener una buena remuneración económica, debe tener al menos dos trabajos. En Colombia, la falta de agenciamiento, por parte de los profesionales en la construcción de las políticas claras para la profesión, es evidente, lo que trae como consecuencia una baja remuneración económica y poco reconocimiento de la profesión como actor en la calidad en el sistema de Salud.

A partir de esta necesidad, en Colombia, con el Decreto 1011 de 2006, que establece el Sistema Obligatorio de Garantía de la Calidad en Salud (sogcs), y en Chile, con la Ley N. ${ }^{\circ} 1$ de 2005, que establece los estándares mínimos de funcionamiento para entidades prestadoras de salud, se busca disminuir el riesgo en la prestación de los servicios y mejorar su calidad, con el propósito de que esta se convierta en cultura y no en requisito $(4,5)$. Este trabajo compara inicialmente los modelos de salud, luego describe el concepto de calidad, posteriormente analiza los estándares de habilitación y acreditación y finalmente sintetiza las similitudes y diferencias de la garantía de la calidad de los dos países.

\section{Materiales y métodos}

Se realizó un análisis comparativo descriptivo entre países (Colombia y Chile), en cuanto a los sistemas de salud y su historia y la caracterización correspondiente a indicadores básicos de salud, con el fin de contrastar los sistemas de Habilitación y Acreditación, específicamente en fisioterapia/ kinesiología. Para esto se utilizó información documental de la normatividad de ambos países, se comparó el Sistema Obligatorio de Garantía de Calidad en Salud para Colombia (Decreto 1011 de 2006) y la Autorización Sanitaria para Chile (Decreto 58 de 2008); se realizaron visitas de campo guiadas a 
entidades hospitalarias de ambos países de alto nivel de complejidad, para verificar por medio de listas de chequeo y de observación directa a los procesos de calidad de los servicios, en relación con los estándares de recurso humano, infraestructura, equipamiento, entre otros, para la Habilitación y Acreditación. Luego, se sistematizó la información recolectada de las normas y las observaciones a las instituciones de las realidades de cada país en materia de requisitos de prestación de servicios para kinesiología.

\section{Marco teórico}

Contextualización de los modelos de salud Las reformas del sector salud en la mayoría de los países de América Latina han mostrado favorecer la participación del sector privado en la gestión pública y la prestación del servicio. Esto muestra una visión neoliberal, que no es otra cosa que la idea de libre mercado.

Algunos factores determinantes extrasectoriales provocaron dichos procesos de reforma, entre los cuales está la crisis del estado de bienestar, la pérdida del rol del estado en garantizar la salud, la crisis de financiamiento de los esquemas de seguridad social, transiciones demográficas que evidencian el envejecimiento poblacional, aumento de las cifras de desempleo en países industrializados y no industrializados, y cambios políticos e ideológicos de comienzos del siglo. En cuanto a factores sectoriales, se evidencia la revolución tecnológica, el aumento del costo de las funciones de producción respecto de la atención en salud y las expectativas de la población para reclamar mejor eficiencia de los sistemas de salud, entre otros $(3,6)$.

Siendo así, países como Chile y Colombia, objeto de este estudio, han llevado varios procesos de reformas para hacer de la salud un derecho fundamental, convirtiéndose esta en el pilar de los sistemas de salud de cada país.
Caracterización Colombia - Chile: El modelo de salud de los dos países está basado en el aseguramiento, Chile posee algunos indicadores con mejores resultados, relacionados con salud como la esperanza de vida al nacer, probabilidad de morir antes de alcanzar los 5 años, probabilidad de morir entre los 15 y 60 años, la tasa de incidencia de la pobreza, proporción de número de camas hospitalarias. En la tabla 1 se describen aspectos sociodemográficos y algunos indicadores sociales relacionados con salud.

Modelo de salud de Colombia: El Sistema General de Seguridad Social en Salud (sgsss) está conformado por dos subsistemas denominados Régimen Contributivo y Régimen Subsidiado. El primero es el encargado de afiliar a trabajadores asalariados, pensionados e independientes con capacidad de pago. El segundo está encargado de afiliar a personas sin capacidad de pago. Existen también regímenes especiales para Fuerzas Militares, Policía Nacional, Ecopetrol y Magisterio.

Todos los Colombianos deben estar afiliados obligatoriamente al Sistema General de Seguridad Social en Salud (sGsss), por intermediación de las Empresas Promotoras de Salud (EPS), que pueden ser públicas o privadas, y son las encargadas de administrar los recursos, afiliar a los usuarios y ofrecer servicios estipulados por la Comisión de Regulación en Salud (CRES); sin embargo, esta entidad fue liquidada el 7 de junio de 2013, obedeciendo al Artículo 2 del Decreto 2560 de 2012, por efecto de la Ley 210 de 2013 en el Plan Obligatorio de Salud (POs), que contiene diferentes servicios según el régimen.

El régimen contributivo está financiado gracias a las cotizaciones de sus afiliados, y el régimen subsidiado está financiado con base en fondos fiscales que provienen del Estado y por aportes del régimen contributivo. 
Tabla 1. Indicadores sociodemográficos de Colombia y Chile

\begin{tabular}{|c|c|c|}
\hline Aspectos & Colombia & Chile \\
\hline Población & 47133782 & 17402630 \\
\hline Extensión & $1141748 \mathrm{~km}^{2}$ & $756946 \mathrm{~km}^{2}$ \\
\hline Sistema Político & \multicolumn{2}{|c|}{$\begin{array}{c}\text { República unitaria, } \\
\text { democrática y multipartidista }\end{array}$} \\
\hline Modelo de Salud & \multicolumn{2}{|c|}{ Aseguramiento } \\
\hline $\begin{array}{l}\text { Tasa de incidencia de la pobreza, sobre la base de la línea de } \\
\text { pobreza nacional (\% de la población) (2011) }\end{array}$ & 34,1 & 14,4 \\
\hline Esperanza de vida al nacer (años) & 75,2 & 79,1 \\
\hline Tasa de natalidad & 18,8 & 14,7 \\
\hline Tasa de mortalidad & 5,8 & 5,7 \\
\hline $\begin{array}{l}\text { Probabilidad de morir antes de alcanzar los cinco años (por } \\
1000 \text { nacidos vivos) }\end{array}$ & 18 & 9 \\
\hline $\begin{array}{l}\text { Probabilidad de morir entre los } 15 \text { y los } 60 \text { años, h/m (por } \\
1000 \text { habitantes) }\end{array}$ & $154 / 76$ & $113 / 58$ \\
\hline Población con discapacidad & $\begin{array}{c}2943971 \\
\text { (Proyección DANE 2012) } \\
\text { Prevalencia 6,3\% }\end{array}$ & $\begin{array}{c}2068072 \\
\text { (FONADIS 2004) } \\
\text { Prevalencia: 6,3\% CASEN } \\
2011\end{array}$ \\
\hline Cobertura en salud & $95,7 \%$ & $96,5 \%$ \\
\hline Camas de hospitalización & 65110 & 31247 \\
\hline Número de egresos hospitalarios (2010) & 1823523 & 1623875 \\
\hline Gasto de bolsillo (2009) & $50 \%$ & $64,6 \%$ \\
\hline Ingreso nacional bruto per cápita (en dólares) & 10110 & 21590 \\
\hline Coeficiente Gini & $57(2010)$ & $52(2009)$ \\
\hline Gasto total en salud por habitante (USD int, 2011) & 618 & 1,292 \\
\hline PIB (USD a precios actuales) (2012) & $369,812,739,540$ & $268,313,656,099$ \\
\hline Gasto total en salud como porcentaje del рів (2011) & 6,1 & 7,5 \\
\hline
\end{tabular}

Datos tomados del Banco Mundial (2013) (7), Salud en las Américas: Panorama regional y perfiles de país (2012) (8), Ministerio de Protección Social (2013) (9), Deparatamento de Estadistica de Información en Salud (2013) (10), Organización Mundial de la Salud (2013) (11).

Dentro del sistema existe una entidad denominada Fondo de Solidaridad y Garantía (FosYGA), el cual se encarga de recaudar los fondos por efecto de cotizaciones y de pagar a las EPS el valor equivalente a la UPC (es la prima que se reconoce a las EPS por cada uno de los afiliados al Régimen Subsidiado y Contributivo, con el fin de garantizar el acceso a los servicios contemplados en el Pos), dependiendo la cantidad de afiliados que tenga cada una de ellas (12). 
Las EPS contratan Instituciones Prestadoras de Salud (IPs), que pueden ser públicas o privadas para proveer la atención correspondiente a la población. Sin embargo, el sector privado ofrece atención a la población de medianos recursos en adelante, incluyendo pago de bolsillo de estos (13).

Modelo de Salud de Chile: Denominado Sistema Nacional de Servicios de Salud (sNSs), está compuesto por el Ministerio de Salud, quien ejerce la rectoría del sistema respecto de la formulación, control, evaluación, actualización de políticas, programas, lineamientos, al igual que la promulgación de normas sobre materias de su competencia. El Ministerio de Salud se encarga de coordinar los Servicios de Salud, el Instituto de Salud Pública, la Central de Abastecimiento, el Fondo Nacional de Salud (FONASA) y la Superintendencia de Salud. El ministerio tiene a su cargo una subdivisión compuesta por la Subsecretaría de Salud Pública, quien es responsable de la administración en materias relativas a promoción, prevención, vigilancia y control de enfermedades, igualmente está encargada de ejecutar acciones de salud pública por medio de Secretarías Regionales Distritales de Salud (SEREMI). La otra subdivisión es la Subsecretaría de Redes Asistenciales, responsable de la articulación y desarrollo de la red asistencial del sistema y de la regulación de la prestación de los servicios de salud de manera mixta (público/privado), ya que la prestación del servicio está conformada por una Red Pública, conformada por hospitales de diversa complejidad, centros de diagnóstico y terapéuticos, centros de referencia de salud y centros de salud de carácter urbano o rural y postas, mientras que la red privada comprende clínicas, centros médicos, laboratorios y farmacias (14).

El sistema es financiado con impuestos generales, contribuciones obligatorias y copagos por medio del fONASA. El sistema tiene su propia Red de Servicios de Salud, y el Sistema Municipal de Atención Primaria; sin embargo, también contrata con el sector privado, también financiado con contribuciones obligatorias mediante las Instituciones de Salud Previsionales ISAPRE. Tanto FONASA COMO ISAPRE son fiscalizadas por la Superintendencia de Salud (3).

En cuanto a accidentes laborales y enfermedades profesionales, son atendidos por mutuales entendidas como seguros adicionales. Las Fuerzas Armadas y Carabineros cuentan con un Sistema Especial de Salud Previsional.

Similitudes de los modelos de salud: Los sistemas de salud en Colombia y Chile se caracterizan por ser modelos mixtos o de competencia gestionada, el Estado desempeña la función rectora y reguladora por medio del Ministerio de Salud. El financiamiento del sector proviene del Estado, cotizaciones de trabajadores, empresas, gasto de bolsillo. Ambos tienen regímenes para poblaciones especiales. El aseguramiento se hace por medio de EPS para Colombia y de FONASA e ISAPRE para Chile. En cuanto lo que representa un aporte a la protección Social en Salud, Colombia cuenta con un Plan Obligatorio de Salud, que es un listado de más de 5000 actividades, procedimientos e intervenciones en salud y servicios hospitalarios, alrededor de 730 o más medicamentos para atención de toda y cualquier condición de salud, enfermedad o trauma para usuarios de cualquier edad afiliados a cualquier régimen. Por su parte, Chile cuenta con la aplicación del Régimen de Garantías explícitas, el cual cubre un grupo de enfermedades cuyo tratamiento está garantizado en cualquiera de los regímenes (15).

Calidad en Salud: La AsQC (Association Society Quality Control) define calidad como un "conjunto de características de un producto, proceso o servicio que le confiere su aptitud para satisfacer las necesidades del usuario o cliente" (2). 
El tránsito de evolución para la calidad puede ser visto en tres aspectos importantes. El primero, a partir de la Revolución Industrial conocido como Calidad Total, el cual consistió en la inspección de productos terminados para aprobarlos o no. El segundo, iniciado a mediados del siglo xx, conocido como control estadístico de procesos, que trataba de la aplicación de técnicas estadísticas para reducir costos. Por último, el Control Total de la Calidad, conocido por sus siglas en ingles TQM (Total Quality Control), introduciendo nuevos conceptos que implican que el control de la calidad es asunto de todos; partiendo de la primicia de que los trabajadores son los primeros empoderados de la responsabilidad de la calidad (2).

Partiendo de esto, en 1951 se crea en Estados Unidos la Joint Comision On Acreditation of Hospitals, organismo encargado de la generalización de las normas de acreditación en los hospitales y las definiciones de los primeros parámetros de calidad en Salud. Posteriormente, en 1961, Donabedian publica el primer artículo relacionado con la calidad en la atención médica, el cual fue pauta para seguir desarrollando conceptos, las nuevas bases de la calidad en la asistencia médica y el control de esta, fundamentada en tres pilares: análisis de la estructura, de proceso y de resultados. La oms ha mostrado su interés por desarrollar la calidad en servicios de salud y, para esto, en los años ochenta empezó a implementar estrategias de procesos de mejoramiento continuo (PMC) que, junto al TQM, están basados en los trabajos de W. Ewards Deming, Joseph Juran, Armand Fiegenbaum y Kaoru Ishikawa, considerados pioneros de la Calidad (16).

La gestión de la calidad se ha convertido en uno de los temas más importantes en el siglo xxI, en especial en temas relacionados con la salud. A partir de esta necesidad, en Colombia, con el Decreto 1011 de 2006, que establece el sistema
Obligatorio de Garantía de la Calidad en Salud (sogcs), y en Chile, con la Ley 1 de 2005, que establece los estándares mínimos de funcionamiento para entidades prestadoras de salud, ambos países, con sus respectivas normas, buscan disminuir el riesgo en la prestación de los servicios y mejorar su calidad, con el propósito de que la calidad se convierta en cultura y no en requisito (4).

Sistema Obligatorio de Calidad de la Atención en Salud, Colombia: Mediante el Decreto 1011 de 2006 se estableció el Sistema Obligatorio de Garantía de Calidad de la Atención de Salud (sogcs) del sgsss. Las acciones a desarrollar de este sistema están orientadas a la mejora de los resultados en salud, centrados en el usuario, cumpliendo con las características de accesibilidad, oportunidad, seguridad, pertinencia y continuidad.

Dentro de sus componentes se encuentran: (a) el Sistema Único de Habilitación, (b) la Auditoria para el Mejoramiento de la Calidad de la Atención de Salud, (c) el Sistema Único de Acreditación y (d) el Sistema de Información para la calidad.

Las entidades responsables del funcionamiento del sogcs son el Ministerio de la Protección Social, como órgano rector; la Superintendencia Nacional de Salud, quien ejerce la función de vigilancia, inspección y control; las Entidades Departamentales y Distritales de Salud, quienes cumplen y hacen cumplir las disposiciones de la reglamentación y las Entidades Municipales de Salud, quienes brindan la asistencia técnica para implementar auditorías a los prestadores de servicios de su jurisdicción (4).

Habilitación: El Sistema Único de Habilitación comprende estándares de obligatorio cumplimiento y son:

[...] el conjunto de normas, requisitos y procedimientos mediante los cuales se establece, registra, verifica y controla el 
cumplimiento de las condiciones básicas de capacidad tecnológica y científica, de suficiencia patrimonial y financiera y de capacidad técnico administrativa, indispensables para la entrada y permanencia en el Sistema, los cuales buscan dar seguridad a los usuarios frente a los potenciales riesgos asociados con la prestación de servicios y son de obligatorio cumplimiento por parte de los Prestadores de Servicios de Salud y las EAPB (17).

Los estándares aplicables son el talento humano, infraestructura, dotación, medicamentos dispositivos médicos e insumos, procesos prioritarios, historia clínica, registros e interdependencia de servicios (18).

Acreditación: El Sistema Único de Acreditación es:

Es el conjunto de entidades, estándares, actividades de apoyo y procedimientos de autoevaluación, mejoramiento y evaluación externa, destinados a demostrar, evaluar y comprobar el cumplimiento de niveles superiores de calidad por parte de las Instituciones Prestadoras de Servicios de Salud, las EAPB y las Direcciones Departamentales, Distritales y Municipales que voluntariamente decidan acogerse a este proceso (19).
Grupos de estándares: Los estándares se encuentran ordenados de la siguiente manera:

- Grupo de Estándares del Proceso de Atención al Cliente Asistencial: derechos de los pacientes, seguridad del paciente, acceso, registro e ingreso, evaluación de necesidades al ingreso, planeación de la atención, ejecución del tratamiento, evaluación de la atención, salida y seguimiento, referencia y contrarreferencia, sedes integradas en red.

- Grupos de Estándares de Apoyo Administrativo-Gerencial: direccionamiento, gerencia, gerencia del talento humano, ambiente físico, gestión de tecnología, gerencia de la información

- Grupo de Estándares de Mejoramiento de la Calidad: Para el caso de Colombia, en la Resolución 1445 de 2006 existe un manual de estándares de Acreditación para las instituciones que ofrecen servicios de Salud de Habilitación y Rehabilitación que son específicos y orientados para las instituciones que ofrecen este tipo de servicios. Para atención al cliente asistencial, los estándares son derechos de los pacientes, acceso, registro e ingreso, evaluación inicial de necesidades al ingreso, planeación de la habilitación / rehabilitación, ejecución de la habilitación / rehabilitación, evaluación de la habilitación / rehabilitación, egreso y continuidad de la atención / integración intersectorial e interinstitucionalidad, y estánda-

Tabla 2. Comparación del Sistema de Habilitación / Autorización Sanitaria

\begin{tabular}{ccc}
\hline Ítem & Colombia & Chile \\
\hline Denominación & Habilitación & Autorización Sanitaria \\
Estándares (Cantidad) & 7 & 4 \\
Obligatoriedad & Sí & Sí \\
Antecedentes & Desde 1979 & Desde 1967 \\
Normativa & Decreto 1011 de 2006 & Decreto 58 de 2008 \\
Órgano rector & Ministerio de Protección social & Ministerio de Salud \\
Vigilancia & Superintendencia Nacional de Salud & Superintendencia de Salud \\
\hline
\end{tabular}


Tabla 3. Acreditación en Colombia y Chile

\begin{tabular}{|c|c|c|}
\hline Ítem & Colombia & Chile \\
\hline Denominación & Acreditación & Acreditación \\
\hline Estándares (Cantidad) & 7 & 9 \\
\hline Obligatoriedad & Voluntario & Voluntario \\
\hline Año de expedición & 2006 & 2007 \\
\hline Normativa & Resolución 123 de 2012 & Decreto Supremo N. ${ }^{\circ} 15$ de 2007 \\
\hline Órgano Rector & Ministerio de Protección Social & Ministerio de Salud \\
\hline Vigilancia & Superintendencia Nacional de Salud & Superintendencia de Salud \\
\hline Entidades acreditadas & 30 & 43 \\
\hline $\begin{array}{c}\text { Relación de entidades acreditadas por } \\
\text { habitantes }\end{array}$ & $\begin{array}{c}1572126 \text { habitantes por } 1 \text { entidad } \\
\text { acreditada }\end{array}$ & $\begin{array}{c}404712 \text { habitantes por entidad acre- } \\
\text { ditada }\end{array}$ \\
\hline
\end{tabular}

res de mejoramiento de la calidad. Los estándares correspondientes a direccionamiento tales como, gerencia, gerencia del talento humano, ambiente físico, gestión de la tecnología, gerencia de la información y los estándares de mejoramiento de calidad son los mismos para todos los servicios. En la tabla 3 se realiza la comparación de los estándares de acreditación (20).

\section{Garantía de Calidad Chile:}

- Autorización Sanitaria: Según el Decreto N. ${ }^{\circ} 58$, el Ministerio de Salud, por medio de las Secretarias Regionales Ministeriales, establece que la autorización sanitaria de los establecimientos asistenciales es obtenida después de verificar el cumplimiento de Normas Técnicas Básicas, que son aplicables a establecimientos públicos y privados. Las Normas Técnicas Básicas de Autorización Sanitaria son aplicables para establecimientos de atención cerrada (Hospitalarios) y abierta (Ambulatorios), tales como hospitalización transitoria, sala para estudios funcionales especiales, anestesia fuera de pabellón, cirugía mayor, imagenología con resonancia magnética, unidades de medicina física y rehabilitación, hospitalización, psiquiatría de corta y mediana estadía, unidades de hemodiálisis, diálisis peritoneal, unidades de quimioterapia, radioterapia, medicina nuclear, unidad de producción de componentes sanguíneos, transporte simple de pacientes, transporte asistido de pacientes (21).

- Requisitos generales establecimiento:ámbito infraestructura, ámbito organización, ámbito recursos humanos y ámbito equipamiento.

El Sistema de Habilitación en Colombia es una herramienta definida para autorizar el ingreso y la permanencia de los prestadores de servicios de salud en el sistema de salud. Está fundamentado en normas sanitarias dirigidas a mantener, restaurar o mejorar las condiciones necesarias en lo relacionado con la salud. Este Sistema tiene como antecedentes la Ley 9 de 1979, que definía la existencia de licencias sanitarias, específicamente para edificaciones en cuanto a la prestación de servicios (22). Hasta el actual concepto de habilitación contenido en el Decreto 1011 de 2006 respecto de la calidad. En Chile, por medio de la Autorización Sanitaria fomenta, protege y recupera la salud de las personas $(23,24)$. Tal autorización es entonces el equivalente, en términos de responsabilidad, en Colombia a la Habilitación, ya que permite a los Prestadores Institucionales de Salud el funcionamiento siempre y cuando cumplan con requisitos de estructura y organización. En la tabla 2 se rea- 
liza la comparación de los sistemas de habilitación y autorización sanitaria entre los dos países.

Acreditación: Dentro del marco de la Reforma de salud se estableció, mediante la Ley de Autoridad Sanitaria N. ${ }^{\circ}$ 19.937, que los prestadores institucionales de salud, tales como hospitales, clínicas, consultorios y centros médicos, para ser acreditados deben cumplir con estándares mínimos de calidad para la seguridad de los usuarios dados por el Ministerio de Salud (24). La Ley N. ${ }^{0} 19.937$ modifica el Artículo $4^{\circ}$ del Decreto Ley N. 2763 de 1979, estableciendo que el Ministerio de Salud tiene la función y potestad de "establecer un sistema de acreditación para los prestadores institucionales autorizados para funcionar", siendo los establecimientos de salud debidamente autorizados los aprobados por los Secretarios Regionales Ministeriales de Salud (23).

Respecto de lo anterior, el Artículo $4^{\circ}, \mathrm{N}^{\circ}{ }^{\circ} 12$, del D.F.L. N. ${ }^{\circ} 1$ de 2005, del Ministerios de Salud, define acreditación como "el proceso periódico de evaluación respecto del cumplimiento de los estándares mínimos, de acuerdo al tipo de establecimiento y a la complejidad de las prestaciones" (25).

Aunque el proceso de acreditación es voluntario para los prestadores, es necesario aclarar que la Ley N. ${ }^{\circ}$ 19.966, que regula el Régimen General de Garantías, al establecer la Garantía Explicita de Calidad, expone dentro de su contexto que las prestaciones de salud deben ser otorgadas por parte de un prestador acreditado ante la Superintendencia de Salud, que en términos concretos indica que para atender patologías GES, es obligatorio estar acreditado (26).

El numeral 11 del actual Artículo $4^{\circ}$ del D.F.L. N. ${ }^{\circ} 1$ de 2005, del Ministerio de Salud, estipula que el Sistema de Acreditación es el mismo para prestadores públicos y privados, y es realizado por entidades públicas o privadas autorizadas por la Superintendencia de Salud (25). En la tabla 3 se compara el sistema de acreditacion de los dos países.

Actualmente, los estándares generales vigentes son el Estándar general de acreditación para Prestadores Institucionales de Atención Cerrada, Estándar general de acreditación para Prestadores Institucionales de Atención Abierta, Estándar general de acreditación para Prestadores Institucionales de Atención Psiquiátrica Cerrada y Estándar general de acreditación para servicios de apoyo no asociados con consultas, ni camas: Servicios de Diálisis, Servicios de Esterilización, Servicios de Imagenología, Servicios de Quimioterapia, Servicios de Radioterapia y Laboratorios Clínicos.

El sistema de acreditación comprende unos ámbitos que son los grandes temas que se evalúan en el proceso. Se expresan como el deber ser de la institución prestadora, respecto de la materia a que este se refiere. Los ámbitos de la acreditación son el Respeto a la dignidad del paciente (DP), Gestión de la calidad (GC), Gestión clínica (GCL), Acceso, oportunidad y continuidad (AOC), Competencias del recurso humano (RH), Registros (REG), Seguridad del equipamiento (EQ), Seguridad de las instalaciones (INS) y Servicios de apoyo (AP) (20).

En la tabla 3 se comparan la acreditación de los dos países con sus respectivos estándares.

\section{Resultados}

Se realizó una matriz comparativa de los estándares de calidad para Autorización Sanitaria / Habilitación (tabla 4) y Acreditación (tabla 5) que se muestra a continuación, donde se evidencia que el sistema de calidad entre los dos países es similar en su estructura y el propósito es ofrecer una atención segura y de calidad. Asimismo, se realiza una descripción y comparación de los estándares y ámbitos en cada uno de los países. 
Tabla 4. Relación de Estándares Habilitación /Autorización Sanitaria

\begin{tabular}{|c|c|c|c|c|}
\hline \multicolumn{5}{|c|}{ Sistemas de Calidad } \\
\hline \multicolumn{2}{|c|}{$\begin{array}{l}\text { Autorización Sanitaria Abierta } \\
\text { (Chile) }\end{array}$} & \multicolumn{2}{|c|}{ Sistema de habilitación (Colombia) } & \multirow[t]{2}{*}{ Relación } \\
\hline Ámbitos & Descripción & Estándares & Descripción & \\
\hline 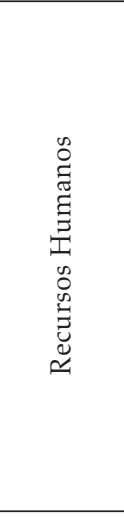 & $\begin{array}{l}\text { - Habilitación } \\
\text { profesional y técnica } \\
\text { - Características del } \\
\text { director }\end{array}$ & Talento Humano & $\begin{array}{l}\text { - Autorización expedida por } \\
\text { la autoridad competente } \\
\text { - Desarrollar acciones de } \\
\text { formación continua de } \\
\text { talento humano en salud } \\
\text { - Determinar la cantidad } \\
\text { necesaria de talento } \\
\text { humano } \\
\text { - Normativa y mecanismos } \\
\text { de control para convenios } \\
\text { docencia - servicio }\end{array}$ & $\begin{array}{l}\text { En Colombia se tienen } \\
\text { en cuenta además } \\
\text { de la autorización } \\
\text { correspondiente de los } \\
\text { profesionales para ejercer } \\
\text { y acciones de formación } \\
\text { continua en talento } \\
\text { humano, los requerimientos } \\
\text { para determinar la } \\
\text { cantidad de profesionales } \\
\text { que necesita un servicio } \\
\text { incluyendo también lo } \\
\text { relacionado con convenios } \\
\text { de docencia - servicio }\end{array}$ \\
\hline 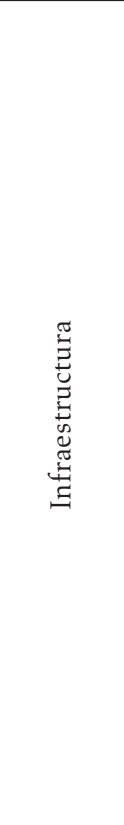 & $\begin{array}{l}\text { - Accesibilidad } \\
\text { - Condiciones } \\
\text { seguridad general } \\
\text { - Recintos generales } \\
\text { - Sala para atención } \\
\text { consulta con examen } \\
\text { - Sala de } \\
\text { procedimientos } \\
\text { - Box de atención de } \\
\text { afecciones respiratorias } \\
\text { - Box de atención } \\
\text { emergencia y } \\
\text { procedimientos } \\
\text { - Sala básica de } \\
\text { rehabilitación } \\
\text { - Aseo } \\
\text { - Recinto disposición } \\
\text { residuos sólidos del } \\
\text { establecimiento }\end{array}$ & Infraestructura & $\begin{array}{l}\text { - Garantía y Condición de } \\
\text { Servicios básicos } \\
\text { - Condiciones de } \\
\text { orden, aseo, limpieza y } \\
\text { desinfección } \\
\text { - Ambientes de trabajo } \\
\text { sucio } \\
\text { - Condición de pisos, } \\
\text { paredes y techos } \\
\text { - Materiales mesones y } \\
\text { superficies de trabajo } \\
\text { - Ascensores y rampas } \\
\text { - Circulación y movilización } \\
\text { de pacientes } \\
\text { - Elementos sucios y } \\
\text { limpios } \\
\text { - Unidades sanitarias para } \\
\text { personas con condición de } \\
\text { discapacidad o movilidad } \\
\text { reducida } \\
\text { - Marco normativo - gestión } \\
\text { integral de los residuos } \\
\text { hospitalarios y similares }\end{array}$ & $\begin{array}{l}\text { Los dos países tienen en } \\
\text { cuenta el ambiente físico } \\
\text { para un buen desempeño } \\
\text { profesional, en el } \\
\text { documento de Autorización } \\
\text { Sanitaria en el ámbito de } \\
\text { infraestructura divide lo } \\
\text { que se debe tener por cada } \\
\text { servicio. En Colombia se } \\
\text { tiene un estándar único } \\
\text { para los diferentes servicios } \\
\text { que incluyen la garantía } \\
\text { y condición de servicios } \\
\text { básicos, además se hace un } \\
\text { especial énfasis en lo que } \\
\text { corresponde a la gestión de } \\
\text { residuos hospitalarios }\end{array}$ \\
\hline
\end{tabular}


Rodríguez-lbagué LF, Sánchez-Medina AF, Zamora-Restrepo PA, Araya-Veliz LA.

\section{Sistemas de Calidad}

\begin{tabular}{|c|c|c|c|}
\hline \multicolumn{2}{|c|}{$\begin{array}{l}\text { Autorización Sanitaria Abierta } \\
\text { (Chile) }\end{array}$} & \multicolumn{2}{|c|}{ Sistema de habilitación (Colombia) } \\
\hline Ámbitos & Descripción & Estándares & Descripción \\
\hline 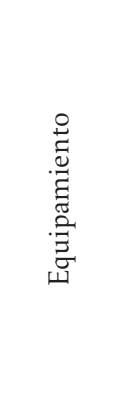 & $\begin{array}{l}\text { - Equipamiento básico } \\
\text { - Carro de reanimación } \\
\text { cardio-pulmonar }\end{array}$ & Dotación & $\begin{array}{l}\text { - Condiciones técnicas de } \\
\text { calidad } \\
\text { - Soportes técnicos } \\
\text { - Unidades sanitarias } \\
\text { - Mantenimiento equipos } \\
\text { - Controles de calidad } \\
\text { - Elementos para } \\
\text { comunicación interna y } \\
\text { externa } \\
\text { - Manual de procedimientos } \\
\text { - Equipos necesarios }\end{array}$ \\
\hline
\end{tabular}

En Chile se incluye equipamiento básico (instrumentos o herramientas propias para realizar una evaluación o examen en una consulta, también describe lo que debe incluir un carro de reanimación cardiopulmonar.

Colombia abarca temas desde los requisitos de las unidades sanitarias hasta condiciones y soportes técnicos de los equipos, mantenimiento, control de calidad, agrega también el requisito de equipos necesarios para realizar diferentes intervenciones dependiendo el tipo de servicio que se preste

Colombia hace especial énfasis en lo que corresponde a seguridad del paciente, cultura institucional, eventos adversos, procesos seguros, riesgo de infecciones asociado con la atención, seguridad en el uso de medicamentos, y guías y protocolos.

- Asignación funciones y responsabilidades - Reglamento interno de seguridad e higiene

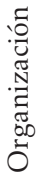
en el trabajo - Reglamentación interna/ manual normas y procedimientos - Documentación reglamentaria - Sistema de registros
- Programa de seguridad del paciente Planeación estratégica de la seguridad

Fortalecimiento de la cultura institucional Medición, análisis, reporte y gestión de los eventos adversos

Procesos Procesos seguros Prioritarios (indicadores de riesgo)

- Detección, prevención y reducción del riesgo de infecciones asociadas con la atención

- Seguridad en la utilización de medicamentos Identificación del paciente en procesos asistenciales - Guías y Protocolos
Chile hace énfasis en documentación reglamentaria, reglamentos de seguridad e higiene en el trabajo. También incluye sistema de registros que sería el equivalente a algunos estándares contenidos en Colombia en historia clínica y registros, y reglamentación interna/ manual, normas y procedimientos que hace alusión a algunos estándares de medicamentos, dispositivos médicos e insumos respecto al manual de acreditación de Colombia 


\begin{tabular}{|c|c|c|c|c|}
\hline \multicolumn{5}{|c|}{ Sistemas de Calidad } \\
\hline Autoriz & $\begin{array}{l}\text { n Sanitaria Abierta } \\
\text { (Chile) }\end{array}$ & \multicolumn{2}{|c|}{ Sistema de habilitación (Colombia) } & \multirow{2}{*}{ Relación } \\
\hline Ámbitos & Descripción & Estándares & Descripción & \\
\hline \multirow{3}{*}{\multicolumn{2}{|c|}{ 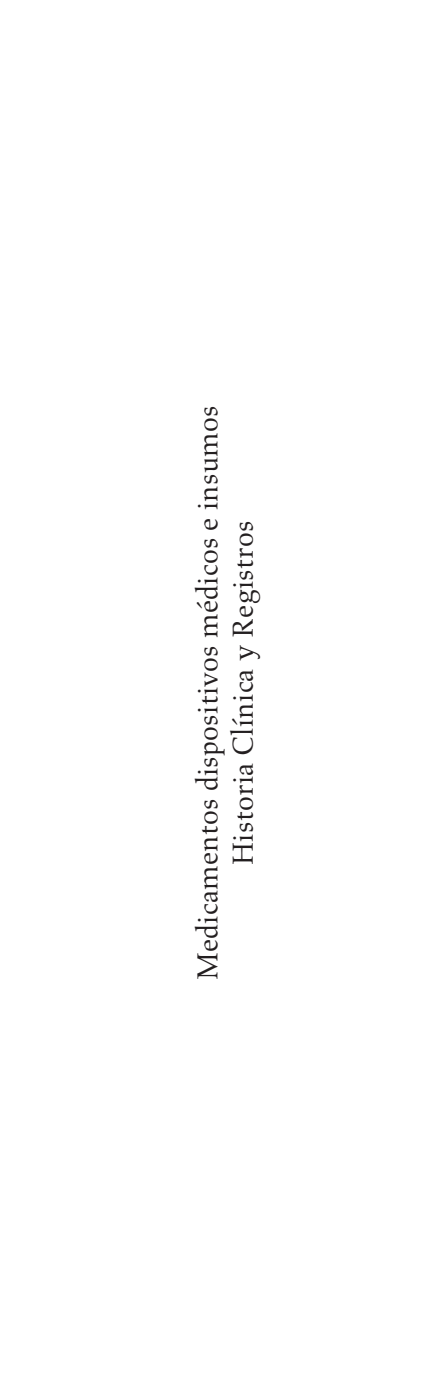 }} & $\begin{array}{l}\text { Interdependencia } \\
\text { de servicios }\end{array}$ & $\begin{array}{l}\text { - Servicios o productos, } \\
\text { propios o contratados } \\
\text { de apoyo asistencial o } \\
\text { administrativo, necesarios } \\
\text { para la prestación de } \\
\text { servicios }\end{array}$ & \multirow[t]{3}{*}{$\begin{array}{l}\text { No se hace alusión en las } \\
\text { Normas Técnicas Básicas de } \\
\text { Autorización Sanitaria }\end{array}$} \\
\hline & & $\begin{array}{l}\text { - Registros - } \\
\text { INvima } \\
\text { - Dispositivos } \\
\text { médicos } \\
\text { - Documentación } \\
\text { especificaciones } \\
\text { técnicas } \\
\text { - Condiciones de } \\
\text { reúso } \\
\text { - Buenas prácticas } \\
\text { de elaboración }\end{array}$ & $\begin{array}{l}\text { Chile no tiene ámbitos } \\
\text { específicos que se refieran } \\
\text { a temas relacionados con } \\
\text { insumos y dispositivos } \\
\text { médicos; solo describe en } \\
\text { el ámbito reglamentación } \\
\text { interna/ manual, normas } \\
\text { y procedimientos. Los } \\
\text { procedimientos de } \\
\text { conservación, custodia } \\
\text { de estupefacientes, } \\
\text { psicotrópicos, y productos } \\
\text { farmacéuticos }\end{array}$ & \\
\hline & & $\begin{array}{l}\text { - Historia Clínica } \\
\text { - Apertura historia } \\
\text { clínica } \\
\text { - Uso de la historia } \\
\text { clínica } \\
\text { - Confidencialidad } \\
\text { y Seguridad } \\
\text { - Diligenciamiento } \\
\text { - Conservación } \\
\text { - Consentimiento } \\
\text { Informado } \\
\text { - Registro } \\
\text { tratamientos } \\
\text { suministrados } \\
\text { - Generación de } \\
\text { residuos }\end{array}$ & $\begin{array}{l}\text { En Chile el ámbito de } \\
\text { sistema de registros incluye } \\
\text { archivo y conservación } \\
\text { de los registros, } \\
\text { confidencialidad, registros } \\
\text { estadísticos, fichas clínicas } \\
\text { individuales, entre otras. } \\
\text { Colombia hace énfasis en } \\
\text { la manera de desarrollar, } \\
\text { diligenciar, utilizar } \\
\text { y destinar la historia } \\
\text { clínica. Tiene en cuenta el } \\
\text { registro de tratamientos } \\
\text { suministrados al paciente } \\
\text { y la relación con el } \\
\text { consentimiento informado. } \\
\text { Incluye también el manejo } \\
\text { de residuos de las historias } \\
\text { clínicas físicas }\end{array}$ & \\
\hline
\end{tabular}


Rodríguez-lbagué LF, Sánchez-Medina AF, Zamora-Restrepo PA, Araya-Veliz LA.

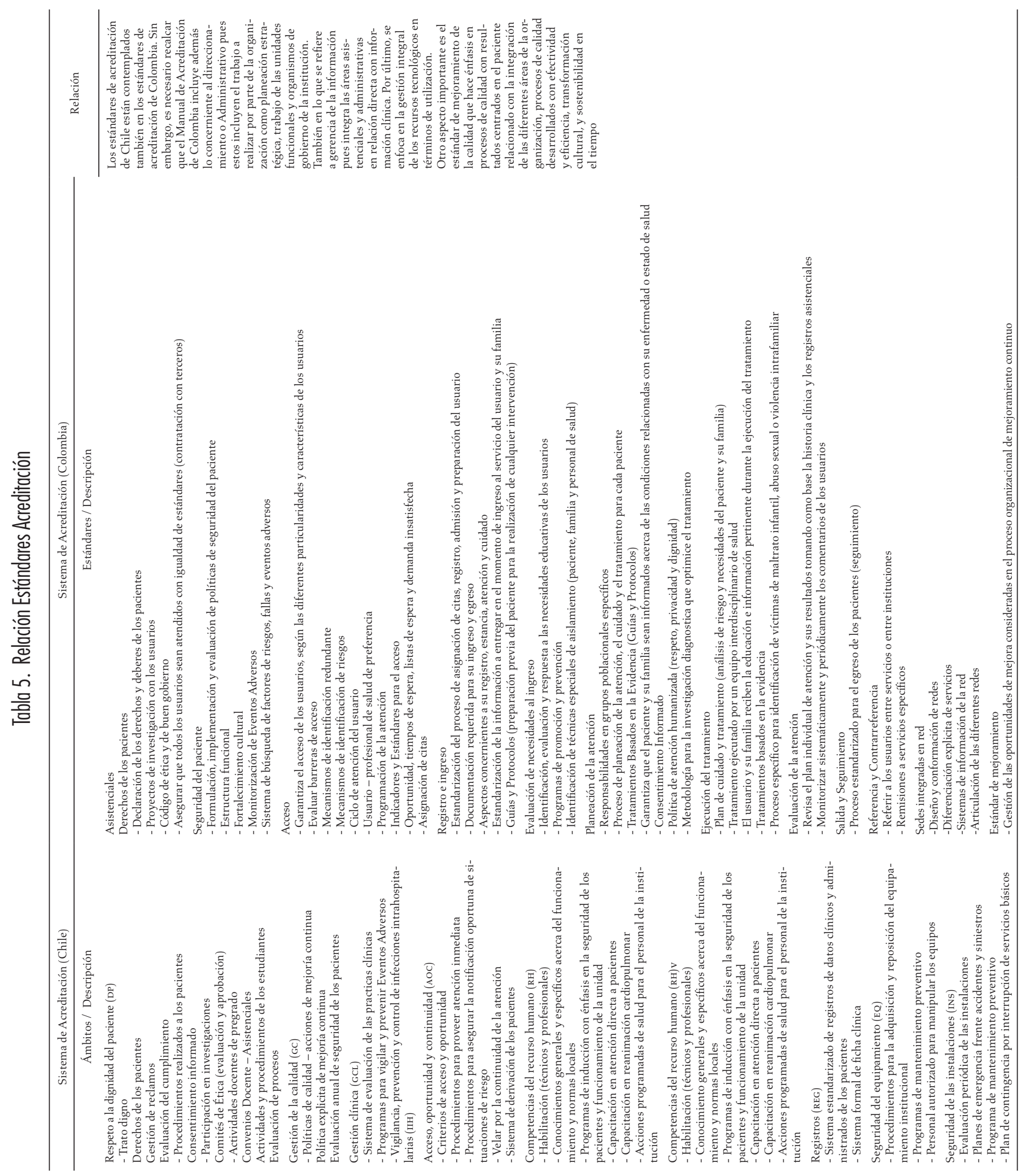


Análisis comparado de los procesos de calidad en fisioterapia / kinesiología de Colombia y Chile

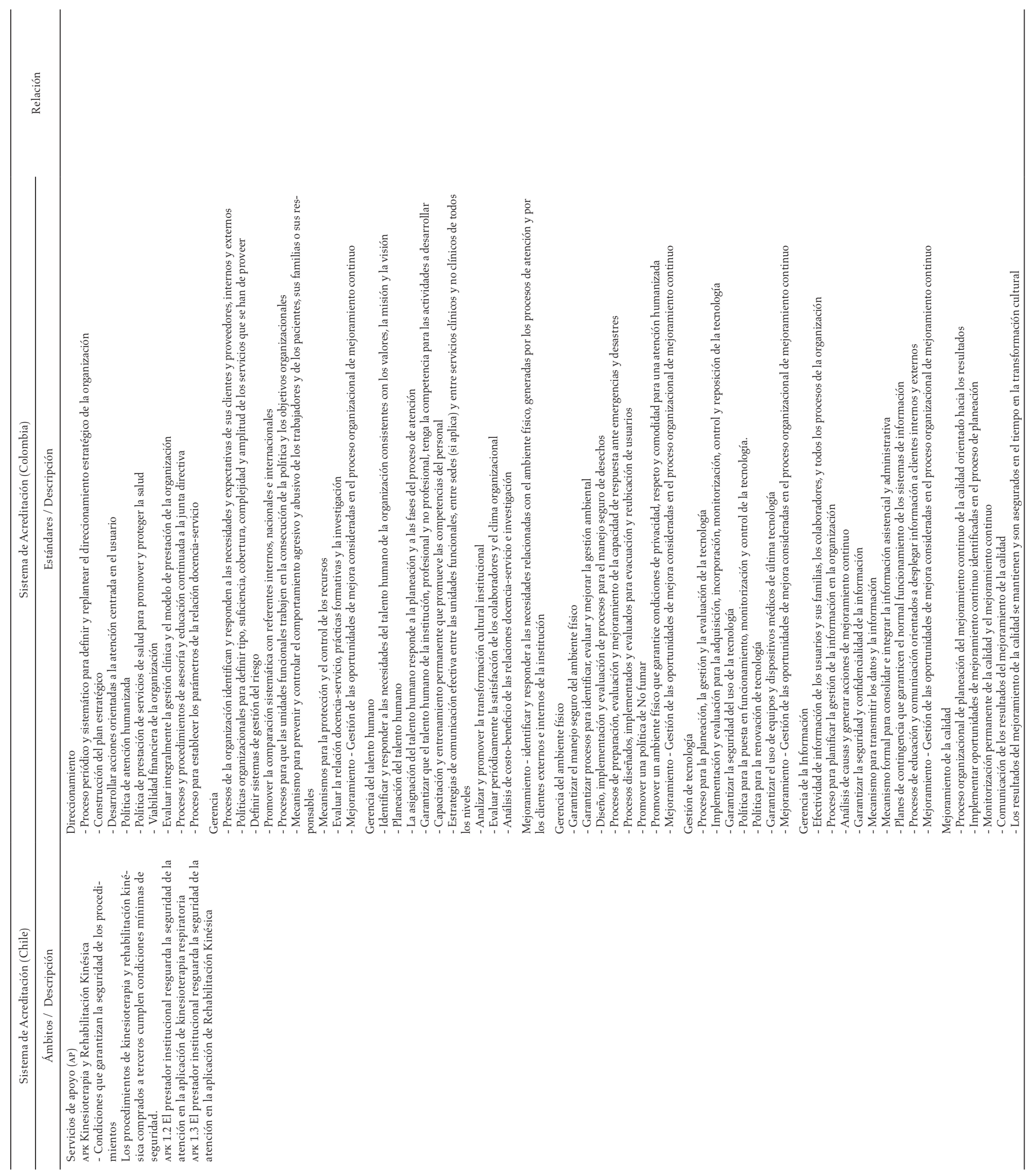




\section{Discusión}

En la revisión documental no se evidenció un estudio cuyas características fueran similares a las que aquí se presentan, algunas muestran comparaciones de los modelos de salud sin profundizar en la habilitación y acreditación en los servicios de kinesiología. Los sistemas de salud en Colombia y Chile se caracterizan por ser modelos mixtos o de competencia gestionada, el Estado desempeña la función rectora y reguladora por medio del Ministerio de Salud. El financiamiento del sector proviene del Estado, cotizaciones de trabajadores, empresas, gasto de bolsillo. Ambos tienen regímenes para poblaciones especiales. El aseguramiento se hace por medio de EPS para Colombia y por medio de FONASA e ISAPRE para Chile. En la protección Social en Salud, Colombia cuenta con un Plan Obligatorio de Salud, el cual contiene un listado de más de 5000 actividades, procedimientos e intervenciones en salud y servicios hospitalarios, alrededor de 730 o más medicamentos para atención de cualquier condición de salud, enfermedad o trauma para usuarios de cualquier edad afiliados a cualquier régimen. Por su parte, Chile cuenta con la aplicación del Régimen de Garantías explícitas, el cual cubre un grupo de enfermedades cuyo tratamiento está garantizado en cualquiera de los regímenes.

Este documento presenta y analiza los modelos de calidad de los países y su evolución. A pesar de tener un sistema que habla de cobertura universal y de prevenir los riesgos en la prestación de servicios, en los dos países existen dificultades en la cultura organizacional para la implementación de la acreditación de servicios. Los dos países cuentan con estándares similares respecto de la Habilitación / Autorización sanitaria y Acreditación. Actualmente, en los países se discuten temas como seguridad del paciente y derechos y deberes de los pacientes, todo en pro de garantizar la calidad del servicio.

En cuanto al desarrollo de la profesión en Chile, se encuentra más empoderada y se consi- dera parte fundamental en el equipo interdisciplinario para alcanzar los objetivos trazados en los procesos de Habilitación y Acreditación y, además, es un actor clave en el sistema de salud. Existen igualmente problemáticas similares en los dos países como las barreras de acceso administrativas, la oportunidad de la atención, el tiempo de la sesión, la cantidad de sesiones y el número de pacientes por profesional.

En ambos países objeto de estudio hay un avance significativo relacionado con la normatividad de Habilitación / Autorización Sanitaria y Acreditación para kinesiología. Sin embargo, en Chile los estándares de habilitación y acreditación son más explícitos en relación con la dotación y la tecnología, definida para la prestación de los servicios. Existen algunos estándares semejantes en los dos países como derechos y deberes, historia clínica y seguridad del paciente, que están en desarrollo actualmente y se han venido incorporando a la cultura organizacional de los servicios.

En Colombia, existen actualmente 30 instituciones acreditadas y en Chile el registro de Prestadores Acreditados a marzo de 2013 sumaba 43 centros asistenciales, entre los que se encuentran 12 clínicas, 5 hospitales públicos, 21 centros de salud ambulatorios privados, un centro de Atención Psiquiátrica, Diálisis, imagenología y 2 laboratorios. En el desarrollo de investigación no se evidenciaron centros específicos de Kinesiología y/o rehabilitación acreditados en los dos países.

Para el avance en los procesos de gestión de la calidad de la kinesiología, es importante la referenciación internacional y poder definir estándares específicos en los diferentes niveles de atención que propendan por mejorar las acciones del profesional en salud pública, la prestación de los servicios y la visibilidad en el sistema de salud.

\section{Agradecimientos}

A la Universidad Mayor, en Santiago de Chile y Universidad del Rosario, en Bogotá, Colombia. 


\section{Referencias}

1. Rodríguez-Ibagué LF. La Viabilidad de la Estrategia de Municipios Saludables. 1. ${ }^{a}$ ed. Bogotá: Universidad del Rosario; 2007.

2. Association Society Quality Control [internet]. 2013 [citado 2013 jul 12]. Disponible en: http://asq. org/index.aspx

3. Granados-Toraño R, Gómez-Montoya MC. La Reforma de los Sistemas de Salud en Chile y Colombia: Resultados y Balance. Rev Salud Publica. 2000;2(2):97-120.

4. Colombia, Congreso de la República, Ministerio de Salud y Protección Social. Decreto 1011 de 2006, (2006 abr 3).

5. Chile MdSGd. Decreto con Fuerza de Ley N. ${ }^{\circ} 1$ Artículo 4, N. ${ }^{\circ} 11,2005$.

6. Arriagada I, Aranda V, Miranda F. cepal Naciones Unidas [internet]. 2005 [citado 2013 jul 5]. Disponible en: http://www.eclac.cl/publicaciones/xml/7/23777/sps114_lcl2450.pdf

7. Banco Mundial [internet]. 2013 [citado 2013 jul 19]. Disponible en: http://datos.bancomundial.org/ indicador/sI.POv.GINI

8. Organización Panamericana de la Salud - Organización Mundial de la Salud. Salud en las Américas, Panorama Regional y Perfiles de País. Publicación Científica y Técnica N. 636 [internet]. 2012 [citado 2013 Jul 8]. Disponible en: http://www.paho.org/saludenlasamericas/index.php?option=com_content \&view=article\&id=9\&Itemid=14\&lang=es

9. Colombia, Ministerio de Salud y Protección Social [internet]. 2013 [citado 2013 jul 8]. Disponible en: http://www.minsalud.gov.co/salud/paginas/indicadoresbasicossp.aspx .

10. Departamento de Estadística de Información en Salud [internet]. 2013 [citado 2013 jul 8]. Disponible en: http://www.deis.cl/indicadores-basicos-de-salud/

11. Organización Mundial de la Salud [internet]. 2013 [citado 2013 jul 3]. Disponible en: http://www.whi. $\mathrm{int} /$ countries/col/es

12. Colombia, Ministerio de Salud y Protección Social [internet]. 2013 [citado 2013 jul 12]. Disponible en: http://www.minsalud.gov.co/Lists/Glosario/AllItems.aspx

13. Colombia, Congreso de la República Ministerio de Salud y Protección Social. Ley 1438. (2011 ene 19).

14. Bastidas SG, Valdivia CG. Reforma de la Salud en Chile; el plan AUGE o Régimen de Garantías Explicitas en Salud (GEs). Su origen y Evolución. Santiago: Boletín Escuela de Medicina U.C. Pontificia Universidad Católica de Chile; 2007.

15. Organización Mundial de la Salud [internet]. 2013 [citado 2013 jul 19]. Disponible en: www.pho.org/ saludenlasamericas/index.php?option=com_content\&view=article\&id=29\&itemid=29\&lang=es

16. Chandia-Vidal Y. Enfermeras Pabellón y Esterilización [internet]. 2006 [citado 2013 jul 12]. Disponible en: www.enfermeraspabellonyesterilizacion.cl

17. Colombia, Congreso de la República, Ministerio de Salud y Protección Social. Decreto 1011, Artículo 6. (2006 abr 3).

18. Colombia, Congreso de la República, Ministerio de Salud y Protección Social. Resolución N. 1441. (2013 may 6).

19. Colombia, Congreso de la República, Ministerio de Salud y Protección Social. Decreto 1011, Artículo 41 (2006 abr 3).

20. Colombia, Congreso de la República, Ministerio de Salud y Protección Social. Manual de Acreditación en Salud Ambulatoria y Hospitalario Colombia Versión 003. Bogotá; 2011.

21. Chile, Gobierno de Chile, Ministerio de Salud. Decreto N. ${ }^{\circ}$ 58. 2008. 
Rodríguez-lbagué LF, Sánchez-Medina AF, Zamora-Restrepo PA, Araya-Veliz LA.

22. Colombia, Congreso de la República. Ley 9. (1979 feb 5).

23. Chile, Gobierno de Chile, Ministerio de Salud. Decreto con Fuerza de Ley 725 (1968, ene 31).

24. Chile, Gobierno de Chile, Ministerio de Salud. Ley 19.937. (2004 feb 24).

25. Chile, Gobierno de Chile, Ministerio de Salud. Decreto con Fuerza de Ley N. ${ }^{\circ}$ Artículo 4. 2005 (2006 abr 24).

26. Chile, Gobierno de Chile Ministerio de Salud. Ley N. ${ }^{\circ} 19.966$ (2004 sep 3). 